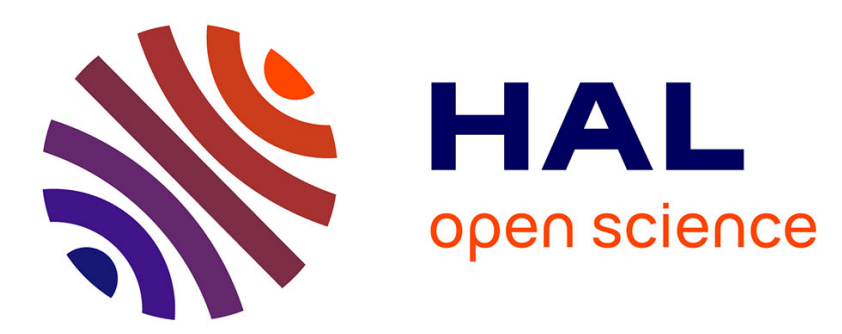

\title{
The Limits of Applicability of Usual Kinetic Relations to the Detonation Waves Chemistry. Homogeneous Explosives
}

B. Kondrikov

\section{- To cite this version:}

B. Kondrikov. The Limits of Applicability of Usual Kinetic Relations to the Detonation Waves Chemistry. Homogeneous Explosives. Journal de Physique IV Proceedings, 1995, 05 (C4), pp.C4-163-C4170. 10.1051/jp4:1995414 . jpa-00253713

\section{HAL Id: jpa-00253713 https://hal.science/jpa-00253713}

Submitted on 1 Jan 1995

HAL is a multi-disciplinary open access archive for the deposit and dissemination of scientific research documents, whether they are published or not. The documents may come from teaching and research institutions in France or abroad, or from public or private research centers.
L'archive ouverte pluridisciplinaire $\mathbf{H A L}$, est destinée au dépôt et à la diffusion de documents scientifiques de niveau recherche, publiés ou non, émanant des établissements d'enseignement et de recherche français ou étrangers, des laboratoires publics ou privés. 


\title{
The Limits of Applicability of Usual Kinetic Relations to the Detonation Waves Chemistry. Homogeneous Explosives
}

\author{
B.N. Kondrikov \\ Mendeleev University of Chemical Technology, 9 Miusskaja Sq., Moscow 125047, Russia
}

\begin{abstract}
The report presented contains the brief description of some of the macrokinetic dependencies in field of detonation of homogeneous, preferably liquid, explosives. Chemical kinetics data and mechanisms of the detonation reactions of pure nitrocompounds at different initial temperature, and the mixtures of them with sulfuric acid are obtained. The data from both groups of measurements for TNT virtually coincide with results of kinetic calculations at which the frequency of spin-pulsating subsurface waves in lean TNT/RDX mixture was used as a source of macrokinetic information. A.N.Dremin theory of failure diameter, usually used for kinetic calculations, is supplemented in three directions. (1) Velocity of the dark wave, one of the most significant elements of the theory, is connected with energy of activation and in this way it is defined independently as an inherent part of the extinguishment process. (2) The gas bubbles formation and growth in the compressed reactive substance in the dark zone are included into consideration as important moments of the detonation wave initiation. (3) The new phenomenon, that has been observed in some of the modern molecular modeling investigations: separation and collection in groups of particles of different mass in shock and detonation waves, was applied in order to explain the strong influence of additives on detonation ability of nitromethane.
\end{abstract}

The usual approximation in physical chemistry of shock waves consists of the suggestion that the thermodynamic equilibria has enough time to establish during the period of the shock wave parameters measurement. If a chemical reaction or polymorph transformation behind the shock front take place, the equilibria can be delayed, and sometimes it should not be taken into consideration at all. Correspondingly kinetic relations have to be taken into account, considered usually however, if one may say so, in so called equilibria approximation: Maxwell-Boltzman energy and velocity distribution is supposed exists, and the relations at least as to the homogeneous systems is concerned do not differ significantly from the usual Arrhenius formulae. The strong acceleration of the chemical transformation in the wave is expected to be a result of the heat evolution, and consequently the heat explosion. A role of pressure, level of which in shock and detonation waves in condensed media is essentially (sometimes in hundreds of thousand, and million times) higher than it appears to be in the routine chemical kinetics investigations, in such a case practically is omitted. 
If the heterogeneous chemical reaction in the wave takes place, in systems from the point of view of density or the phase composition nonhomogeneous, the reaction is originated in sites having the higher temperature or reaction ability level, and it develops from them in form of the relatively slow burning. Due to the high pressure influence it has been proceed mainly in gas phase, and correspondingly the pressure dependence of burning rate can be considered in frames of the usual $u(p)$ law.

The most significant contradiction as to the heterogeneous systems detonation is concerned consists in that the reaction rate estimated by means of the extrapolated burning velocity data does not conform with reaction rate obtained by means of the modern theory of the curved detonation front calculations. As a rule the extrapolated velocity is about 3-6 times less than the data derived from the detonation experiments. It is necessary to introduce some additive suggestions, say influence of pressure oscillations in the reaction zone, the frequency of which is about several GHz. Another possible reason of the explosive fast burning is the fragmentation of bubbles in the incident shock wave.

One can say that the experimental facts and laws in field of the heterogeneous systems detonation that are related by the very complicated way to the real mechanisms of the chemical reaction can hardly give the essentially positive information about the chemical reaction kinetics. Correspondingly they might be only scarcely used as a tool for the problem formulated solution. The homogeneous detonation characteristics provide in this respect more favorable information because they are appeared to be connected with the mechanisms of the reaction at detonation more closely.

The abundant information about kinetics and mechanisms of the detonation reactions in homogeneous and heterogeneous systems was obtained in this Laboratory during the last two decades mainly using the failure diameter - initial temperature, or additives concentration relations, and detonation velocity - charge diameter dependencies. [1-7], and A.N.Dremin and J.Bdzil [8,9] theories application, as well as by means of the shock wave pressure - induction period dependencies [10] treatment. R.Engelke and J.Bdzil [11] had derived kinetic data on nitromethane decomposition in detonation wave from the detonation front curvature measurements. Quite recently a group of investigators of Washington State University [12] using the modern spectroscopy instruments possibilities has obtained interesting data concerning kinetics of nitromethane/ethylenediamine mixture reactions in temperature and pressure region very close to the dark zone at extinguishment of detonation. All the more interesting perspective appears to be the application in the nearest future of pico-, and femtosecond spectroscopy measurements $[13,14]$.

In case of the homogeneous, mostly liquid explosives the $D(d)$ relations as a rule may not be measured accurately due to strong temperature dependence of the reaction rate and the detonation instability influence. Correspondingly, the main source of information on chemistry in the detonation wave from the macroscopic point of view is the failure diameter, $\mathrm{d}_{\mathrm{f}}\left(\mathrm{T}_{0}, \mathrm{C}_{\text {solv. }}\right)$ change, and the classic Dremin theory application. The most amusing fact in this respect is that namely instability in such a case provides the chemical kinetics from $d_{f}\left(T_{0}, C_{\text {solv }}\right.$. $)$ data extraction owing to the peculiar kind of the pulsating reactor formation, in the reaction zone of which the process has developed in the quasi steady state conditions suitable for the kinetic measurements.

We have obtained the initial temperature - failure diameter dependencies for nitromethane, nitroglycol, diethyleneglycoldinitrate, TNT/ $\mathrm{H}_{2} \mathrm{SO}_{4} 50 / 50$ and nitromethane/ $\mathrm{H}_{2} \mathrm{SO}_{4} 50 / 50$ mixtures $[3,5,6]$, and have used the Belyaev and Kurbangalina data [15] for pure TNT and nitroglycerine. The most amazing result is the absence of difference in detonation ability of the liquid and crystal nitroglycol (Fig. 1). It is a check of the idea of possible influence of the crystallic structure of a substance on reaction rate at shock wave attack [16]. The calculations performed demonstrate in particular a good correlation between the data on 
the adiabatic explosion period $\tau(p)$ dependence [10] and our data estimated from $d_{f}\left(T_{0}\right)$ line for NM [7]. The lines $\tau(p)$ obtained for pure NM at different initial temperatures intercept the curve calculated from $d_{f}\left(T_{0}\right)$ relation at approximately the same $T_{0}$ values (Fig.2).

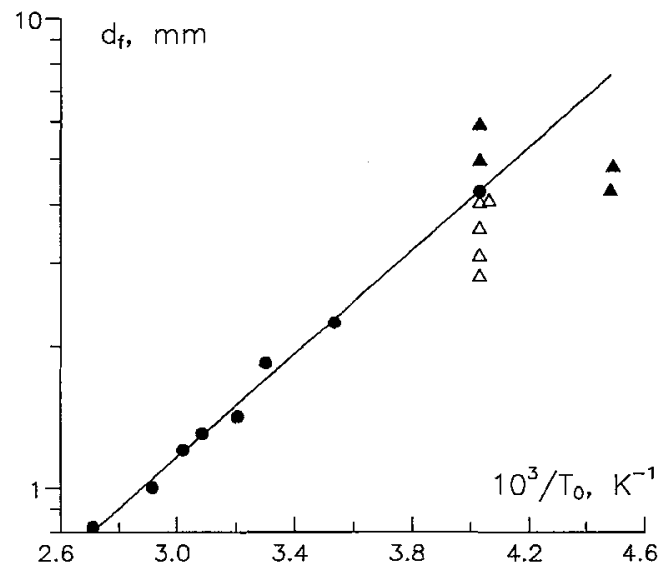

Fig.1. Data on the failure diameter - $1 / \mathrm{T}_{0}$ dependence obtained by G.D.Kozak and V.V.Kondratiev for liquid $(\bullet$, in the conical glass tubes), and crystallic solid $(\Delta, \Delta$. detonation, failure detonation; in the cylinder glass tubes) nitroglycol.

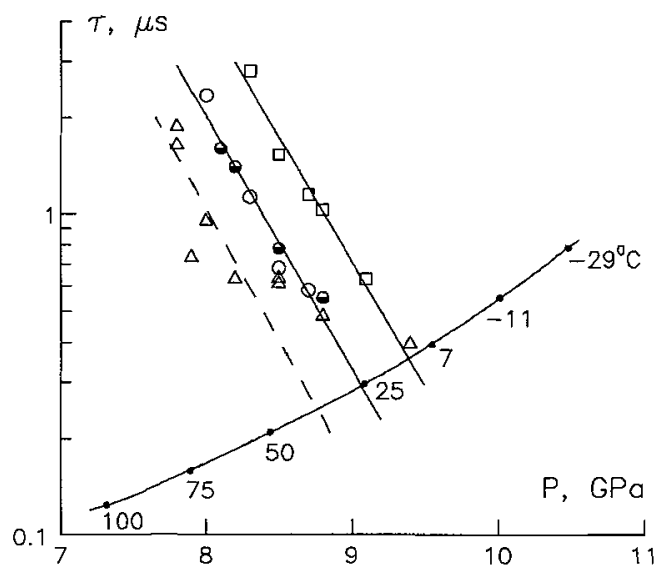

Fig.2. Adiabatic ignition period $\tau$ obtained at the different initial temperatures by the methods: the curve $-\mathrm{d}_{\mathrm{f}}\left(\mathrm{T}_{0}\right)[5]$, the straight lines $-\tau(\mathrm{P})[10]$. $\mathrm{T}_{0}: \Delta 40^{\circ} \mathrm{C}, \quad 00 \quad 25^{\circ} \mathrm{C}, \square \quad 4-6^{\circ} \mathrm{C}$ (gas chromatografically purified and spectral quality NM data only). This figure is taken from [7].

Several years ago a new phenomenon in field of detonation of homogeneous liquid and solid explosives and propellants was observed [4]. At detonation of the cylinder charge, diameter of which is a little bit more than the failure diameter of the substance the spin detonation wave can arise under and near the surface of the charge in a thin layer of the explosive compressed by the main coaxial detonation wave. The velocity of the spin wave may reach $\sim 10 \mathrm{~km} / \mathrm{s}$, and the corresponding high pressure forms a flow of the detonation products and the original material particles which leave the deep spiral furrows on the metal witness plate. The subsurface layer can arise spontaneously owing to the extinguishment of the detonation reaction on the edge of the charge, or it may be formed coercively by cutting preliminary the thin spiral line on the cylinder surface. In case of the spontaneous spin wave formation the phenomenon is supposed to be described as the spin-pulsating process, frequency of which might be used for estimation of the adiabatic self-heating induction period, and correspondingly, the kinetic constants of leading chemical reaction. The thermodynamic conditions in the compressed subsurface layer can be estimated using relations of Dremin's dark wave theory. As a matter of fact the results for TNT demonstrate unity of all the values calculated by different methods, on base of experimental results measured by the quite different ways: $d_{f}\left(T_{0}\right)$ dependence [15], $d_{f}\left(C_{\mathrm{H} 2 \mathrm{SO} 4}\right)[5,6]$, and spin frequency - detonation rate relations give $\mathrm{E}_{\mathrm{a}}=109-110 \mathrm{~kJ} / \mathrm{mol}, \mathrm{k}_{0}=10^{9}-10^{10} \mathrm{~s}^{-1}$. 
Two main uncertainties of Dremin theory should be taken into account before kinetic results might be obtained inherently on the one hand and independently of the more complicated measurements on the other.

The first of them is the uncertain value of the dark wave, or the dumping reaction wave velocity $v$, which in frames of the theory must be determined experimentally.

We have calculated [17] the dark wave velocity in the suggestion, that the detonation front, at least on the edge of the charge, has a form of a wave

$$
y=A \cdot \sin (k \cdot x) \cdot \cos (\omega \cdot t)
$$

where $\mathrm{t}$ is time, $\mathrm{A}$ is amplitude, $\omega=2 \pi / \tau$ is cyclic frequency, $\tau$ is the period of the wave, $\mathrm{k}=2 \pi / \lambda$ is the wave number, $\lambda$ is the length of the wave. In this approximation $v=\lambda / \tau$.

One can suppose that the reaction extinguishment condition is reached, when temperature in the wave becomes less than $T_{*}=T-R \cdot T^{2} / E_{a}$ and $W_{*}=W / e$, where $T$ and $W$ are the steady-state detonation front temperature and reaction rate, $T_{*}$ and $W_{*}$ are critical values of the temperature and reaction rate, $E_{\mathbf{a}}$ is the energy of activation.

At $\mathrm{T} \sim \mathrm{p} \sim \mathrm{D}^{2}$ we obtained

$$
v^{2}=\left(\mathrm{A}^{2} \cdot \omega^{2} / \mathrm{RT}\right) \cdot \mathrm{E}_{\mathrm{a}}
$$

or

$$
v^{2}=a \cdot \mathrm{E}_{\mathrm{a}}
$$

where $a$ is a constant ( $\mathrm{mol} / \mathrm{kg}$ ), which may be defined from the well known data for NM and TNT failure diameter vs. initial temperature relations:

$$
a=65 \mathrm{~mol} / \mathrm{kg} \text {. }
$$

Using this value of $a$ we calculated the more precise data of the overall kinetic constants of the reactions in the dark zone of the extinguishing detonation wave [17].

The second contradiction in Dremin theory consists in the fact that reaction in the dark zone develops in accordance with the adiabatic heat explosion laws: the explosion springs up in the end of the zone as a result of the reaction heat acceleration. However just near the zone a thick layer of the hot - up to $3000 \mathrm{~K}$ gaseous products exists, which moreover is constantly mixing with the compressed initial substance owing to the turbulence stimulated by the difference (about $100 \mathrm{~m} / \mathrm{s}$ ) in velocities of the layers. The second circumstance that should be comprehended in this respect is the mechanisms of deflagration originated from the hot source, whether it be a selfignition or by the hot gas inflammation, to detonation transition.

One way or another, we came to conclusion, that the main process in the dark zone is formation of the gas bubbles, the presence of which was proved some twenty years ago by F.Walker and R.Wasley [18] and then confirmed by some others [19]. When the bubbles concentration and dimensions become high enough to provide the DDT process development in the zone, it will be obtained, originating from the every source which one can propose - the adiabatic selfignition, the inflammation by the hot gas, or due to the influence of the weak shock waves irradiated by the main detonation front oscillations. In any event, the point of the violent reaction initiation should be placed at the end of the dark zone, where the bubbles have enough time to grow to the dimensions necessary for the violent, explosive deflagration, and for the deflagration to detonation transition. 
The process of the bubbles grow can be stipulated by the coalescence of small gas nucleus in the quickly moving turbulent flow, but it seems plausible that it can be connected also with burning of the initial substance after the dimension of the initial "germ" becomes sufficient for inflammation. This dimension is $\sim \mathfrak{x} / \mathrm{u}$, where $\mathfrak{x}$ is the thermodiffusivity coefficient and $\mathrm{u}$ is the linear burning rate.

At usual magnitudes of $\mathfrak{x}$ and $\mathrm{u}$, about $10^{-7} \mathrm{~m}^{2} / \mathrm{s}$, and $1-10 \mathrm{~m} / \mathrm{s}$, correspondingly, we obtain the critical nucleus dimension $\sim 10-100 \mathrm{~nm}$. During induction period of about 0.1-1 $\mu$ s the nucleus can grow up to $2 \cdot \mathbf{u} \cdot \tau \sim(2-20) \mu \mathrm{m}$. These dimensions are quite sufficient for detonation at relatively low shock wave attack initiation, and for the DDT process development. The expansion of the explosive in the dark zone behind the oblique shock wave promotes the grow of the bubbles.

Fortunately, this addition to the model of A.N.Dremin virtually does not affect at least in the first approximation the calculation scheme existed. Formation of the necessary quantity $(\alpha \sim 10 \% \mathrm{v} / \mathrm{v})$ of the gas bubbles needs depending on the extent of expansion of $2-5 \%$ of the initial substance decomposition. It is only about two times more than the theory of adiabatic heat explosion utilized in Dremin model requires. The preignition development of the reaction requires about the same time as the burning of the explosive around the nucleus.

So the uncertainty of about two for the Arrhenius preexponential factor should be anticipated. In this respect the rather strange result of ours [20] concerning the influence of aeration of diglycoldinitrate on the failure diameter of the substance can be explained. Instead of more or less fast decrease of $d_{f}$ at porosity rise as it traditionally is in case of all the other explosives studied, the DGDN's $\mathrm{d}_{\mathrm{f}}$ quickly enhances from about $15 \mathrm{~mm}$ at zero porosity up to at least $50 \mathrm{~mm}$ at porosity of about $5 \%$. The most probable reason of this unusual behavior, strange as it may seem, is the high reactivity of DGDN as a nitric ester. Even a small concentration of bubbles in the liquid leads to the quick burning in the dark zone from the very beginning of the zone formation. The burning destroys the zone as the necessary element of the lateral detonation wave providing the main detonation front regeneration, and in such a strange manner leads to the failure diameter augmentation. The paradoxical character of this explanation consists in that the substance of higher reactivity can have under certain circumstances the higher failure diameter than the more inert one.

The results of our investigations of the "inert" solvent on $d_{f}$ influence $[1-3,5]$ have demonstrated that almost no really inert solvent exists. Oppositely to the wide spread ideas of the past, all of the organic liquids used react intensively with the nitrocompounds or with the products of the decomposition thereof in detonation wave as if they were a part of a molecule of the explosive. Moreover, most of them accelerate the chemical reactions at detonation, consequently decreasing $d_{\mathrm{f}}$. Bases, acids, unsaturated compounds, halogen containing substances, and even distilled water can accelerate the detonation wave reactions (at least in case of NM).

The most impressive of the results obtained is the strong enhancement of the detonation ability of nitrocompounds at mixing them with bases and acids. From the practical point of view especially significant is the unusually strong $d_{f}$ of TNT decrease when adding of the concentrated or superconcentrated, containing excess of $\mathrm{SO}_{3}$, sulfuric acid. The failure diameter of $\mathrm{TNT} / \mathrm{H}_{2} \mathrm{SO}_{4} / \mathrm{SO}_{3}$ mixture reaches approximately $2 \mathrm{~mm}$, some thirty times less than the pure TNT $d_{f}$ value. It is about the failure diameter that have been obtained for such dangerous nitric esters as nitroglycerin or nitroglycol. The theory of the process is proposed in the works $[6,7,21]$.

From the theoretical point of view of course the most essential fact is the catalytic activity of small (up to $0.02 \%$ ) admixture of amines or acids to nitromethane [1,2]. 
The idea proposed at discussion the results with Ja.B.Zel'dovich was the chain reaction initiated by amine or acid. It is necessary to note that this idea needs the suggestion of 150-200 steps long chains in the reaction zone (sometimes during the period of time up to $10^{-6}-10^{-8} \mathrm{~s}$ ). If we take into account that the chain reactions at the nitrocompounds decomposition or detonation in no single one case were definitely observed the idea as a whole becomes at least a very suspicious one. We still had developed it in many details $[3,22]$ in spite of the natural resistance of the authors to the ideas of others. This purely psychological opposition supposedly led us to the new model of the phenomena and to the more pronounced understanding of the processes proceed in the dark zone.

Of course the new concept needs in turn some additive proposals. One of them consists in the fact one could note at the reports of D.Robertson, P.Haskins, J.Boris, E.Oran and some others presented on the IVth Gordon Conference, June 26-30 1994, New Hampton, NH, USA and on the 2nd International Workshop on Microscopic and Macroscopic Approaches to Detonation, Saint Malo, France, October 2-7, 1994. At molecular modeling of detonation forming two different sorts of molecules in products, one has clearly seen that the products are separated: some of them combine the groups, meanwhile the others form a layer around them. This process, which could be called as coalescence in two-phase media, and is appeared to be a peculiar "antidiffusion" in case of the single-phase substance, probably is connected with the strong collisions between the molecules in shock wave. The molecules of the different masses acquire at collisions the different velocities, for instance the more light particles are rejected, thrown away, and the more heavy ones are collected together. Dr.A.A.Borisov has mentioned in private discussion that the process reminds him the well known fact of polarization in shock wave, i.e. separation of ions and electrons due to quick diffusion of electron gas [23]. One cannot exclude however that this is just superficial resemblance of two similar but in the essence still different effects.

If in the dark zone behind the shock wave, formed at initiation or at extinguishment of detonation, the molecules of nitromethane and an additive form a heavy intricate complex, they can combine together into ensemble the dimensions of which are enough to produce the hot spot from which burning of the substance may originate. Correspondingly the system can produce the number of bubbles which is sufficient for the DDT process occurrence at relatively small number of the molecules added.

The nucleus of about $10 \mathrm{~nm}$ in diameter can be formed by $10^{2}-10^{3}$ of the sufficiently big molecules. In this respect, one could mention that the only heavy, big molecules of organic amines are the additives that usually are utilized as the catalysts (though may be mainly from the practical point of view due to involatility of the heavy amines). The concentration of the bubbles of the final dimension of $\sim 10 \mu \mathrm{m}$ originated from the nucleus at the gas relative volume in liquid of about $10 \%$ is $\sim 2 \cdot 10^{14} \mathrm{~m}^{-3}$. It is quite reasonable value. If we propose that number of heavy complexes in one group is about $10^{2}-10^{3}$, then the whole number of the molecules of the catalyst gathered in groups is $2 \cdot 10^{16}-2 \cdot 10^{17} \mathrm{~m}^{-3}$. At the suggested content of amine molecules in nitromethane $\sim 0.02$ mole $\%$ the whole number of the catalyst particles is $\sim 2 \cdot 10^{24} \mathrm{~m}^{-3}$. So the part of the molecules gathered in the groups is only $10^{-5}-10^{-6} \%$ of all the molecules of the additive in the mixture.

This may be rather complicated mechanism of the catalytic additive on nitrocompounds detonation influence has been confirmed however by the results obtained recently by the group of investigators [12] using the methods mentioned above. At essentially isentropic compression of nitromethane containing $0.1 \%$ ethylenediamine up to $13.7 \mathrm{GPa}$ they could observe only first order consumption of $\sim 0.1 \%$ of nitromethane without any significant self-acceleration. Obviously, in absence of the shock-wave collection the amine-nitromethane complexes being not possible to combine in groups decompose separately without forming nucleus of self-ignition and subsequent burning.

This is very important experiment. It shows that $0.1 \%$ ethylenediamine in absence of the shock wave influence do not accelerate nitromethane decomposition process significantly, all the catalyst added 
"burns out" without significant self-acceleration of the nitromethane reaction (although some enhancement of the nitromethane consumption at pressure from 9.8 to $13.7 \mathrm{GPa}$ augmentation is still definitely observed). In any case the consumption of $0.1 \%$ of the explosive itself at the conditions of isentropic compression can give only the very confined, about 3-4 K, temperature rise, and may not lead to the adiabatic self-acceleration. It is necessary to add also that the results [12] actually show that the pressure itself is in no case the determining factor of the heat evolution at reaction in detonation wave.

One can see, that most of the rather unusual effects and dependencies related to the detonation reactions kinetics and mechanisms may be explained quite logically by means of the usual macrokinetic relations drawing the relatively small number of the additive suggestions. Again, one can see that the molecular dynamic calculations results and the modern spectroscopic measurements are extremely useful for understanding of some of the well-known but badly explained facts. It obviously is the main practical output of the heavy and expensive works, development of which is stipulated by the concentrated financial support of several highly qualified scientific groups, and the main purpose of which in turn is namely to use the modern theoretical and experimental methods possibilities for explanation of the great mass of information accumulated during the many decades of the energetic materials shock and detonation phenomena investigation.

\section{REFERENCES}

1. Kondrikov B.N., Kozak G.D., Raykova V.M. et al., Dokl. Akad. Nauk SSSR, 233 (1977) 402-405 (Russ.).

2. Kondrikov B.N., Chemical kinetics of detonation in some organic liquids. 8th ICOGER, Minsk 23-26 August 1981, Shock Waves, Explosions and Detonations, J.R.Bowen, N.Manson, A.K.Oppenheim, R.I.Soloukhin, Eds. (Vol. 87 of Progress in Astronautics and Aeronautics, 1983), pp. 426-441.

3. Starshinov A.V., Cand. Sci. (Ph.D.) Thesis, Mendeleev Institute of Chemical Technology, Moscow, 1979.

4. Kozak G.D., Kondrikov B.N., Oblomsky V.B., Comb., Expl., Shock Waves, 25 (1989) 459-465.

5. Khoroshev S.M., Cand. Sci. (Ph.D.) Thesis, Mendeleev Institute of Chemical Technology, Moscow, 1987.

6. Gamezo V.N., Kozak G.D., Kondrikov B.N. et al., Explosive Materials and Pyrotechnics, CNINTIKPK (1992), N 3 (218), pp.3-9 (Russ.).

7. Gamezo V.N., Diploma (Master's Degree) Thesis, Mendeleev Institute of Chemical Technology, Moscow, 1988. Cand. Sci. (Ph.D.) Thesis, Mendeleev Institute of Chemical Technology, Moscow, 1992.

8. Dremin A.N., Dokl. Akad. Nauk SSSR, 147 (1962) 870-873 (Russ.).

9. Bdzil J.B., J. Fluid. Mech. 108 (1981) 195-226.

10. Berke J.G., Shaw R., Tegg D. et al., "Shock initiation of nitromethane, methylnitrate, and some bisdifluoramino-alcanes", V Symp. (Int.) on Detonation, Pasadena, Ca, 18-21 August, 1970 (ACR-184, Office of Naval Research, 1971) pp.237-246.

11. Engelke R., Bdzil J.B., Phys. Fluids, 26 (1983) 1210-1221.

12. Constantinou C.P., Winey J.M., Gupta Y.M., J.Phys.Chem. 98 (1994) 7767-7776.

13. Lee I.-Y.S., Hill J.R., Dlott D.D., J. Appl. Phys., 75 (1994) 4975-4983. 
14. Wang W., Weferss M.M., Nelson K.A., "Femtosecond spectroscopy of chemical reactive solids: a methodology", Structure and Properties of Energetic Materials, Boston, Ma, USA, 30 November - 2 December, 1992. D.H.Liebenberg, R.W.Armstrong and J.J.Gilman Eds. (MRS, Piltsburgh, Pa, 1993) pp.130-140.

15. Belyaev A.F., Kurbangalina R.H., Journ. Fiz. Khim., 34 (1960) 603-610 (Russ.)

16. Kondrikov B.N., "The kinetics of chemical reactions in physico-chemical waves", Ya.B.Zel'dovich Memorial, Moscow 12-17 September, 1994, S.M.Frolov, Ed. (Russian Section of the Combustion Institute, Vol. 2, 1994) pp.36-38.

17. Gamezo V.N., Kondrikov B.N., Khimicheskaya Fizika, 13 (1993) 1502-1505 (Russ.).

18. Walker F.B., Wasley R.J., Comb. Flame, 22 (1974) 53-67.

19. Vorobjev N.A., Trofimov V.S., Fizika Gorenija i Vzryva, 14 (1978) 152-153.

20. Kondrikov B.N., Kozak G.D., Oblomsky V.B. et al., Comb., Expl., Shock Waves, 23 (1987) 195-202; "Peculiarities of detonation of porous liquid nitrocompounds", Fundamental Problems of Physics of Shock Waves, Azau, i 8-21 May, 1987, (Acad. Sci. USSR, Vol. 1, part 1, 1987) pp. 34-36.

21. Gamezo V.N., Khoroshev S.M., Kondrikov B.N. et al., "Sulfuric acid influence on the nitrocompounds detonation reaction", 2nd Workshop on Macroscopic and Microscopic Approaches to Detonation, Saint Malo, France, 2-7 October, 1994.

22. Starshinov A.V., Kondrikov B.N., Kozak G.D. "Homogeneous catalysis at nitromethane detonation", I Vsesoyusnoye Sovestchaniye po Detonatsii, Chernogolovka, 1977. (Detonation, Akad. Sci. USSR, Chernogolovka, 1977), pp. 73-76.

23. Zel'dovich Ya.B., Raizer Yu.P., Physics of shock waves and high temperature hydrodynamic effects ("Nauka", Moscow, 1966), p.404. 


\section{2-3 Discussion}

Moderator: Dr. Gabrielle Dupré, C.N.R.S., Laboratoire de Combustion des Systèmes Réactifs (LCSR), Orléans, France

In the session dedicated to detonation in liquids and gases, some new ideas, experiments and calculations have been presented, concerning the initiation and propagation of detonation in homogeneous media, the threedimensional structure and its relationship to chemical kinetics, the energy release involved during the detonation process, the initiation of detonation in a liquid explosive. Then, statements from two laboratories gave some insight over the validity of the classical theories.

Afterwards, an interesting discussion took place between the audience and the speakers. Various questions have been posed or comments stated, as you can see below, concerning GZND theory, critical parameters for the initiation phase of detonation, wave curvature, strong initiation, properties of matter above the critical point, location of the onset of chemical reactions in liquid explosives, anisotropic properties and polarization effects within the shock front in liquid explosives...

In the following pages, I have tried to restitute the discussion as fair as I could, according to the cassette, with some slight modifications in order to take into account what the authors wrote afterwards.

\section{A.N. Dremin A comment:}

The CJ detonation theory has not proposed any mechanism of EMs transformation at detonation and therefore one should not expect from the theory the detonation limits (SDT, unstability, failure diameter) and interpretation. In regards to the ZND theory it has appeared in fact as some response to the need for the theory capable to interpret the limits. The notion of the finite chemical reaction zone at detonation is undoubtly the main point of the EMs transformation mechanism proposed by the ZND detonation theory.

The following experimental findings are inconsistent with the ZND detonation theory: First: Very small change of liquid EMs detonation velocity $D$ with charge diameter $d$ near their rather big failure diameter $d_{f}$. Opposite to this finding, it follows from the theory that any EMs $D(d)$ dependence and their $d_{f}$ have to correlate; the smaller is the dependence -- the smaller $d_{f}$ has to be.

Second: The fact that explosion induction time at shock initiation of detonation in homogeneous (gaseous and liquids) EMs becomes negligibly small at a shock pressure approximately equal to CJ pressure and not at the pressure of the shock of the CJ detonation velocity.

Third: Unstable detonation. Before the ZND detonation theory advent, one believed that one-head and many-headed spinning detonations were separate original phenomena different from normal detonation. Later on, one tried to interpret the instability as some small perturbations of the ZND detonation wave physical model. However, it turned out that the unstable detonation physical model and the ZND detonation model have nothing common in general. They differ fundamentally.

In essence the theory could not explain the unstable detonation origin mechanism. The nature elicitation has led to a new phenomenon discovery. The phenomenon has come to be known as chemical reaction breakdown. It has been revealed that all limits of detonation are governed by the phenomenon. 
phenomenon has come to be known as chemical reaction breakdown. It has been revealed that all limits of detonation are governed by the phenomenon.

\section{Clavin-Desbordes: about critical length and critical energy}

Clavin: What are the mechanisms involved in the critical length and critical energy, in the initiation phase of detonation? In few words, I would say that, as shown by Desbordes, there is a critical radius below which it is not possible to have a self-sustained detonation. This can be explained by non-linear dynamic effects that may occur. It is not surprising that there is a natural length scale which is the induction length. It is clear that below this length, you cannot have any spherical detonation. This was the argument of Zel'dovich, even in his papers. But what is surprising in the experiments is that the ratio between the critical radius Desbordes measured and the induction length is about 600 to 700. Now, if you go through the critical energy, that is $\mathrm{r}_{0} \mathrm{D}_{\mathrm{CJ}}{ }^{2} \mathrm{~L}_{\mathrm{iCJ}}{ }^{3}$, the number that we have to explain is no more 700 but $700^{3}$, that gives a number which is between $10^{6}$ and $10^{8}$. This is a real question that we have to answer.

Desbordes : For me, the induction length $\mathrm{L}_{\mathrm{i}}$ is just a concept derived from the model of detonation called ZND. This length remains nevertheless very instructive for the detonation point of view. The only relevant and real length, which is due to a more complex detonation structure than the ZND model, is the cell size $\lambda$, typical length of a three-dimensional structure, which is surprisingly proportional to $\mathrm{L}_{i}$, which is a onedimensional length. As a consequence, no conceptual reason forbids the existence of a detonation in a tube of diameter $d$, equal to or of the same order as $L_{j}$. Unfortunately, such a detonation is not observed. Detonation is only observed if $d=\lambda_{C J}$. A similar observation is found for curved detonation where a criterion for the existence of detonation has to be discussed between the radius of curvature and the cellular structure, that is the more elementary micro-scale of detonation that can be found.

Oran : about the improvement of reaction scheme and its influence on detonation cell size

I would like to comment only the effects of the chemical reactions on the size of the detonation cells. Just recently, we have done a series of computations on $\mathrm{H}_{2} / \mathrm{O}_{2}$ reaction scheme. We went from a rather crude reaction scheme for $\mathrm{H}_{2} / \mathrm{O}_{2}$ which models just an induction time and an energy release to an improvement of the chemistry, using $2 \mathrm{D}$ calculations and a four chemical kinetic reaction-scheme. We found that, in fact, as we made this improvement of the chemistry, we got significant effects of the size of the detonation cell; this is because of the distribution of the energy release.

\section{Kondrikov-Desbordes : about intense source of explosion}

Kondrikov: What did you say about the nature of the dark zone around the exploding wire? Is it just a strong detonation and a reaction zone too narrow to irradiate light, or is it an effect of expansion of the evaporated metal ? Can you measure the velocity in this zone ? How big is it?

Desbordes : The energy released by an intense source of explosion (as the exploding wire initiation source) creates in gases a very strong spherical expanding shock wave which decays as it expands. Generally, in a reactive medium, an overdriven spherical detonation is created and, if the characteristic chemical length (i.e. the post shock chemical induction length of the global reaction) is too large in comparison to the radius of curvature when the detonation approaches the $C J$ regime of the wave, then the 
detonation vanishes completely. So, in a general way, a CJ self-sustained detonation cannot resist to a too strong curvature of the wave compared to its chemical induction length.

Melius-Presles, Desbordes, Dremin : about properties of gases and liquid above the critical point

Melius : My question is about gases and liquids both, because at high pressures and temperatures, above the critical point, we cannot anymore distinguish the gas from the liquid. So it seems to me that perhaps the differences are due to the density rather than to the properties of the liquid or the gas and, unfortunately, the issues arisen at high temperature and pressure may not be appropriate terms anymore and, if you find many similarities, it is perhaps because of the density that makes the difference.

Presles : I do not understand very well the question, you are talking about differences between what and what?

Melius : We do not held the concept of a liquid or a gas anymore above the critical point, they are just the same fluid. So what are the differences between a liquid and a gas that we are trying to differentiate like in low an high density materials ? What effects of detonation of gases versus liquids might be attributable to differences in density rather than in phase characteristics?

Presles : It is a large question.

Desbordes : Detonation in either gas or liquid phase gives detonation products above the critical point. For gases, the EOS of ideal gas can generally be applied. For liquid explosives, when pressure effects are very high because pressure in detonation products is proportional to initial density, we need an EOS of the detonation products that eventually takes into account some solid species. Chemical kinetics may be certainly be completely different, even if there are both homogeneous media.

Dremin : May I comment this question? You know that even at a very high range of pressures, it is a real liquid. In all our shock experiments, we are under the critical point. If you put a text at the bottom of a vessel of a liquid explosive and run a shock in it, you can read the text through the shock compressed material. The transparency of shock compressed materials is extremely nice.

\section{Dremin : about rate of energy release and rate of adiabatic cooling}

I would like to continue with the following problem. As a matter of fact, whatever is the shape of the wave, a planar or a spherical one, it does not matter. The fact that the shock transforms into a detonation or not depends on two parameters : the rate of energy release and the rate of adiabatic cooling. If the adiabatic cooling cannot compensate the rate of energy release, then the shock turns into detonation. If the rate of adiabatic cooling can compensate completely the release of energy, then the shock cannot transform into detonation.

Boris-Dremin, Nelson, Gupta : about anisotropic properties of liquid explosives Boris : My question is to all of you who have worked with liquid explosives. In the Navy, we deal a lot with water and it is understood that water can have very anisotropic properties on short time scales. We are talking about very fast shock and initiation of detonation in liquids. Do any of these liquids display some of the anisotropic properties that we have talked earlier, these structure-based anomalies usually associated with 
solids, such as anisotropy, dislocations, holes, etc ? Or do liquids behave more homogeneously like dense gases?

Dremin : In comparison with water that looses its transparency at some specific pressure, liquid explosives do not change their transparency under the shock pressures usually used for the initiation of detonation. That means that they change their chemical structure very slightly, in comparison to water. Liquid explosives behave like dense gases until shock intensities induce a critical pressure able to initiate a detonation.

Nelson : Anisotropy is another question that will go away from the liquid on a time scale of few picoseconds. It may last longer if there exist some cavitations or some macroscopic structures.

Gupta : You can have rotational molecules in a liquid, although it is believed that molecular rotation occurs very rapidly, at a 30-50 ps time scale. However, under a pressure of 50-60 kilobars, it is not clear that it can happen within picoseconds time scale. We have some spectroscopic observations which we have interpreted as to be due to the rotation of molecules under shock conditions.

Coming back to the issue about anisotropy, there is a very interesting paper of Tony Campillo where he looked at life-times, and it turns out that if we shock something, on the average we go very quickly to anisotropic conditions.

Dlott-Presles, Ramsay : about relationship between beginning of chemistry and region of slight discoloration

Dlott : You showed pictures of the initiation of liquid nitromethane. They show the dark regions and the light regions and, in between, there is a slight discoloration region and you pointed out and said : this is where the chemistry began. How do you know there was not lots of chemistry before that, which simply did not appear in your picture ?

Presles : You are right : on the picture, we see luminosity and we say that chemical reactions start at that time, but in any case, chemical reactions have begun earlier. It is because this is the only way to measure some induction time and it is why I propose (and not only me) to use an electrical method which may more precise.

Ramsay : In response to the question on the reaction in shocked liquids, basically the first reference to that was given in a conference which was held around 1963 ; also in the last Detonation Symposium, there are several papers that have addressed that question. There are reactions which starts relatively early, with an energetics apparently very low, because it is very low detectable in fact in the shock front. So we have argued basically for simplicity in saying that the reaction starts when we see the light. But in fact, there is some sort of reaction which occurs earlier. And the light is quite strong in liquid TNT and in other liquid explosives.

Dremin-Presles, Dremin, Presles : about electrical polarization in the shock front

Dremin : You are talking about electrical polarization in the shock front. What do you mean ? Are explosive molecules dipoles or is there a deformation of molecules in the shock discontinuity zone, then an interaction of the shock with the deformed molecule?

Presles : I do not know exactly what happens inside the shock front, but I only refer to previous work on polarization effects in polar molecules, which says that this effect is due to the orientation of dipoles inside the medium. So whatever the medium, explosive or not, when it is submitted to a shock wave, we get this effect and, in this case, the medium seems to be anisotropic, because it appears that there is an orientation of some dipoles. 
Dremin : Sometimes, we observe polarization signals in reflected shock wave, because under the first shock, the molecule is very deformed and there is some separation of charges.

Presles : But we do not observe any electrical signal at the first passage of the shock wave. I agree with you.

Melius-Borisov: about the two-stage heat release

Melius : I think that in the two-stage heat release, you must convert nitro-compounds to $\mathrm{NO}$ and $\mathrm{NO}$ to $\mathrm{N}_{2}$. You are observing some other characteristics of materials that may absorb.

Borisov : I was talking about the heat release kinetics which is important for the detonation phenomena. Because of the heat release, the mixture is heated, temperatures rises, the reaction is accelerated and this acceleration just removes the second stage because, at the end of reaction, its rate is so high that you just do not see the second stage. You have a smooth heat release rate, up to the final temperature. But in real experiments, we do observe the second stage. 